\title{
Bioconversion of Gamma-Aminobutyric Acid from Monosodium Glutamate by Lactobacillus brevis Bmb5 ${ }^{\text {s }}$
}

\author{
Anna Jeong ${ }^{\dagger}$, Cheng Chung Yong ${ }^{\dagger}$, and Sejong $\mathrm{Oh}^{*}$ \\ Division of Animal Science, Chonnam National University, Gwangju 61186, Republic of Korea
}

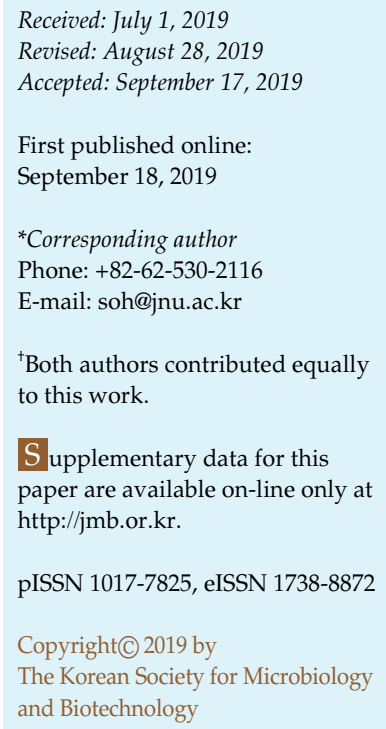

Gamma-aminobutyric acid (GABA) plays important roles in host physiology. However, the effects of GABA are greatly restricted due to its low bioavailability in the human body. Here, a high acid-tolerance GABA-producing strain, Lactobacillus brevis Bmb5, was isolated from kimchi. Bmb5 converted glutamate to GABA $(7.23 \pm 0.68 \mu \mathrm{g} / \mu \mathrm{l})$ at a rate of $72.3 \%$. The expression of $g a d B$ gene, encoding the enzyme involved in the decarboxylation of glutamate to GABA, was decreased upon incubation. Our findings indicate GABA production in Bmb5 is not directly correlated with gadB gene expression, providing new insight into the mechanisms underlying GABA production in Lactobacillus.

Keywords: Monosodium glutamate, Lactobacillus, GABA, gene expression, glutamic acid decarboxylase
Gamma-aminobutyric acid (GABA) is a non-protein amino acid with several important physiological functions, including mitigating hypertension, diabetic-vascular, and neurological disorders [1-3]. However, the bioavailability of naturally occurring GABA and the restriction on synthetic GABA is limiting its beneficial effects in humans. Increasingly, the use of lactic acid bacteria (LAB), which is generally regarded as safe (GRAS) with numerous health benefits, have emerged as a feasible approach to improve the bioavailability of GABA in food $[4,5]$. To date, several GABA-producing LAB strains have been identified, including Lactobacillus bucheneri [6], Lactobacillus brevis [7, 8], and Lactobacillus plantarum [9]. These high GABAproducers are generally found in acid-based fermented foods [7], suggesting traditional fermented foods could be a potential source for novel LAB strains. Therefore, in this study, we mined the high GABA-producing LAB from kimchi, a Korean traditional fermented food.

A total of 26 Lactobacillus strains were isolated from homemade traditional kimchi in Gwangju, Jeollanam-do, Korea and maintained in de Man, Rogosa, and Sharpe
(MRS) medium (Difco, USA) at $37^{\circ} \mathrm{C}$ (data not shown). Due to the association between acid tolerance and GABA production, the LAB isolates were first screened for their ability to tolerate acid [10, 11]. The activated lactobacilli cultures were incubated in acidic MRS (pH 2.5 with $1,000 \mathrm{U} / \mathrm{ml}$ pepsin) at $37^{\circ} \mathrm{C}$ for $2 \mathrm{~h}$ and the viability was then determined [12]. As a result, only eight out of 26 isolates, including the LAB strains $5,11,12,13,16,18$, J1, and $\mathrm{Bmb} 5$, possessed a prominent ability to tolerate acid. No significant decreases $(\rho<0.05)$ in the number of viable cells were observed in these eight strains after the acid challenge (Table S2). It has been reported that glutamic acid decarboxylase (GAD) system, a biosynthesis system that maintains the intracellular $\mathrm{pH}$ homeostasis in LAB, can contribute to the acid tolerance and GABA production in LAB strains. GAD has been shown to be associated with $\mathrm{F}_{1}-\mathrm{F}_{0}$ ATPase, agmatine deiminase (AgDI), and arginine deiminase (ADI) system. It improves the transmembrane electrical potential and consumes intracellular protons during the decarboxylation of glutamate for the production of GABA, thereby contributing to the acid tolerance and 


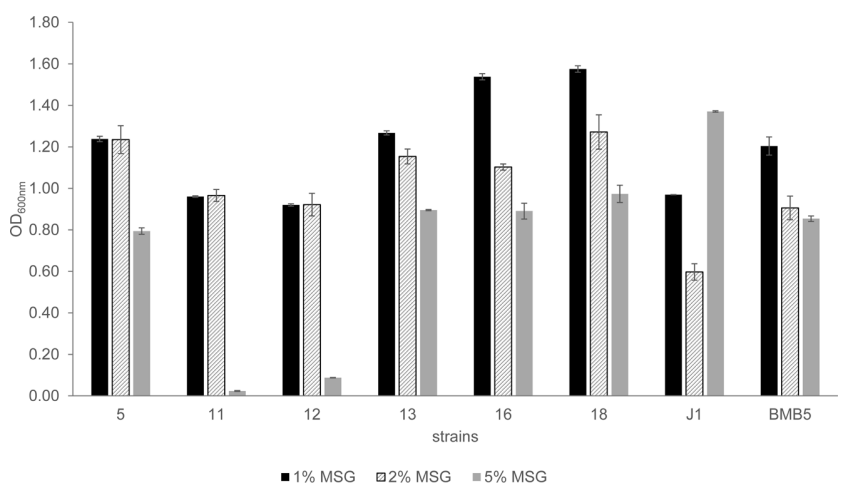

Fig. 1. Effect of different concentration of MSG on the growth of Lactobacillus strains. Lactobacillus strains were grown in MRS media supplemented with $1 \%, 2 \%$ or $5 \%(\mathrm{w} / \mathrm{v})$ of MSG at $37^{\circ} \mathrm{C}$ for $18 \mathrm{~h}$, and optical density at $600 \mathrm{~nm}$ was recorded. Results are expressed as the mean \pm standard deviation from three independent experiments. Black bar, 1\% MSG; shaded bar, 2\% MSG; grey bar, 5\% MSG.

restoring the intracellular $\mathrm{pH}$ homeostasis of $\mathrm{LAB}[10,11$, $13,14]$. It is therefore implicated that LAB strains with high acid tolerance could also be the potential high-GABA producers.

Next, we used monosodium glutamate (MSG), a common food additive, as a substrate to elucidate the production of GABA in the selected LAB strains. All strains remained viable upon incubation in MRS media containing $1 \%(\mathrm{w} / \mathrm{v})$ MSG. However, the growth of certain strains was suppressed in the presence of $2 \%$ and $5 \%(\mathrm{w} / \mathrm{v})$ of MSG in the media (Fig. 1). To this end, MSG at the concentration of $1 \%(\mathrm{w} / \mathrm{v})$ was used for subsequent analyses.

To investigate the capability of these LAB strains to convert MSG to GABA, we then determined the levels of GABA upon incubation via GABAse assay, an enzymatic detection method as previously described [15]. While all strains were capable of producing a significant amount of GABA, ranging from 2.22 to $7.23 \mu \mathrm{g} / \mu \mathrm{l}$, L. brevis Bmb5 exerted the highest GABA production $(7.23 \pm 0.68 \mu \mathrm{g} / \mu \mathrm{l})$, with a bioconversion rate of $72.3 \%$ from MSG to GABA. Its capability to convert MSG to GABA was highly comparable with the high GABA-producing reference strain, L. brevis KCTC $13094(7.43 \pm 0.60 \mu \mathrm{g} / \mu \mathrm{l})$ [16]. Several studies have reported that the species of LAB and Bifidobacterium isolated from acid-based fermented foods produced a similar concentration of GABA [17, 18]. Additionally, we also obtained similar findings in the HPLC analysis, with both L. brevis Bmb5 (713 ppm) and KCTC 13094 (720 ppm) had the highest GABA production,
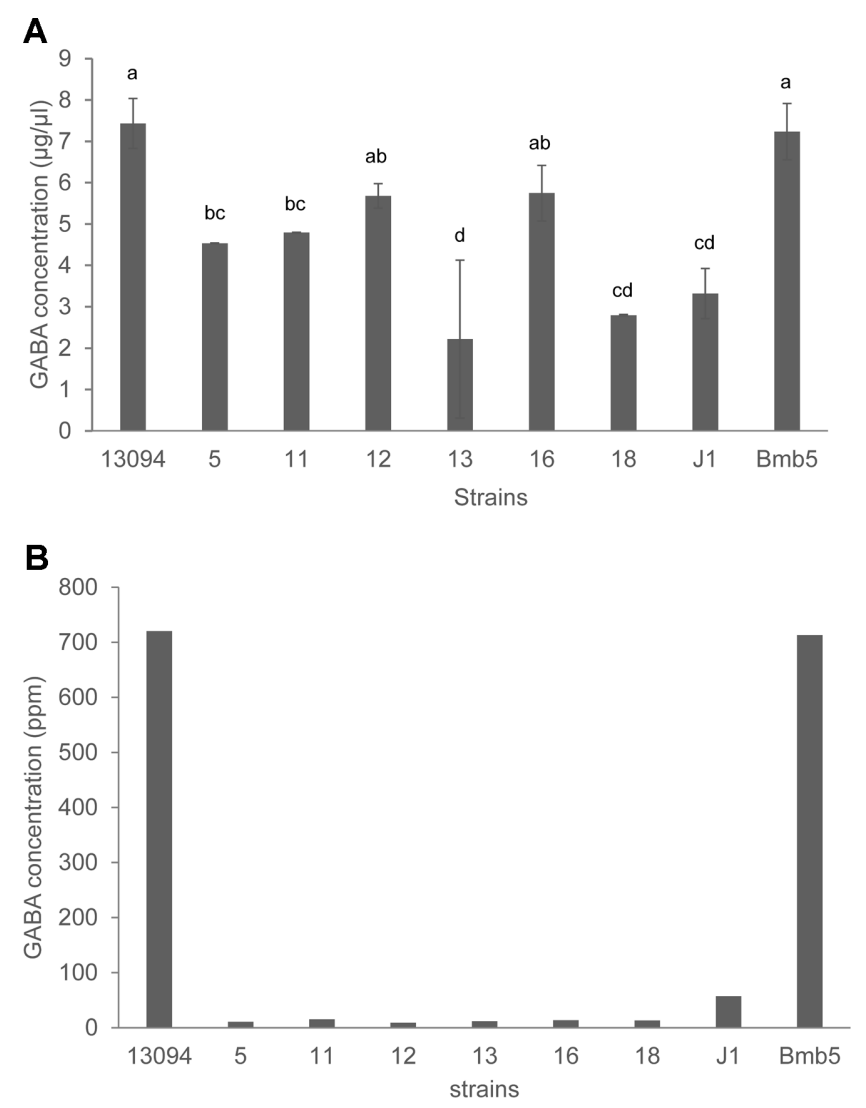

Fig. 2. GABA production by Lactobacillus strains in the presence of $1 \%(\mathrm{w} / \mathrm{v})$ MSG measured via (A) GABAse assay and (B) HPLC.

Results are expressed as the mean \pm standard deviation from three independent experiments for GABAse assay; Data from one of the repetitions are shown herein for HPLC analysis. Tukey's multiple range test was performed for GABAse assay, with difference lowercase letters $(a, b, c$, and $d)$ demonstrated the significant differences between samples $(\rho<0.05)$.

as compared to the other strains.

As stated earlier, GABA is known to be produced via the GAD system, which consists of $\operatorname{gadB}$ and gadC genes. Lglutamic acid is transported across the membrane via the glutamate/GABA antiporter $(\mathrm{gadC})$ and irreversibly $\alpha$ decarboxylated into GABA through the enzymatic action of glutamic acid decarboxylase ( $g a d B)$, which is localized in the cytoplasm of most GABA-producing $\operatorname{LAB}[10,13]$. Previous studies have reported that the activity of GAD was maximum at acidic conditions ( $\mathrm{pH} 4-5)$ [14, 19]. In this study, the expression level of the $\operatorname{gadB}$ and $\operatorname{gadC}$ genes in the GAD system of L. brevis Bmb5 and L. brevis KCTC 13094 was determined using qPCR.

As shown in Fig. 3, the gene expression of gadB and gadC 

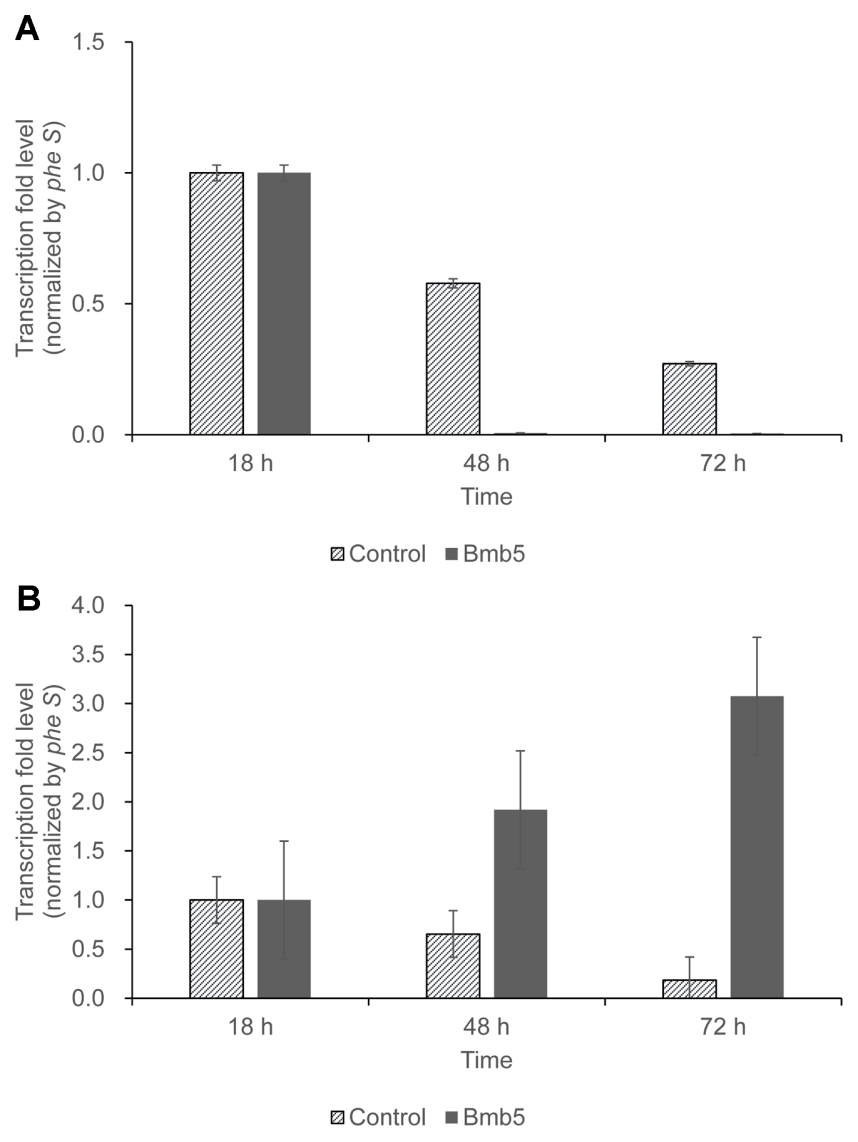

Fig. 3. Relative gene expression of (A) $g a d B$ and (B) $g a d C$ of GAD system of Lactobacillus brevis Bmb5 in the presence of $1 \%$ (w/v) MSG.

Results are expressed as the mean \pm standard deviation of three independent experiments. Shaded bar, control strain, Lactobacillus brevis KCTC 13094; grey bar, Lactobacillus brevis Bmb5.

was initially unaffected (at $18 \mathrm{~h}$ ). The expression of $\operatorname{gadB}$ gene was decreased after $18 \mathrm{~h}$, where the cells began to deteriorate, as shown in the growth curve of L. brevis Bmb5 (Fig. S1). GABA is produced via the decarboxylation of glutamate, catalyzed by the glutamic acid decarboxylase which encoded by the gadB gene [10, 13]. Recent studies reported that the gene expression of $g a d B$ was unaffected by glutamate, suggesting high GABA production is attributed to the catalytic activity of GAD instead of the biosynthesis of GAD [20-22]. Our findings showed that GABA production was not directly correlated to the gene expression of the $\operatorname{gadB}$, indicating the mechanisms underlying GABA production in L. brevis Bmb5 is yet to be discovered. In contrast, the gene expression of gadC was gradually increased over time, indicating the glutamate/ GABA antiporter remains active during the later stage to actively transport the GABA produced and glutamate across the membrane, thereby contributing to the intracellular $\mathrm{pH}$ homeostasis and high acid tolerance in L. brevis Bmb5.

The use of GABA-producing LAB offers an alternative approach to counter the restriction on the use of synthetic GABA and the low bioavailability of naturally-occurring GABA in foods. The findings from this study indicate that the high GABA-producing L. brevis Bmb5 isolated from kimchi could be potentially used for developing GABAenriched foods for promoting health or for pharmaceutical purposes. In addition, the correlation between the GAD system and the bioconversion of MSG to GABA provide new insight into the discovery of mechanisms underlying the GABA production.

\section{Acknowledgment}

This work was supported by the Basic Science Research Program through the National Research Foundation of Korea (NRF) funded by the Minister of Education, Science, and Technology (NRF-2016R1A2B4007519 and NRF-2019R1A2C108764811).

\section{Conflict of Interest}

The authors have no financial conflict of interest to declare.

\section{References}

1. Jakobs C, Jaeken J, Gibson K. 1993. Inherited disorders of GABA metabolism. J. Inherit. Metab. Dis. 16: 704-715.

2. Adeghate E, Ponery A. 2002. GABA in the endocrine pancreas: cellular localization and function in normal and diabetic rats. Tissue Cell. 34: 1-6.

3. Wong CGT, Bottiglieri T, Snead OC. 2003. Gaba, $\gamma$ hydroxybutyric acid, and neurological disease. Ann. Neurol. 54: S3-S12.

4. Seok J-H, Park K-B, Kim Y-H, Bae M-O, Lee M-K, Oh S-H. 2008. Production and characterization of kimchi with enhanced levels of $\gamma$-aminobutyric acid. Food Sci. Biotechnol. 17: 940-946.

5. Leroy F, De Vuyst L. 2004. Lactic acid bacteria as functional starter cultures for the food fermentation industry. Trends Food Sci. Technol. 15: 67-78.

6. Cho YR, Chang JY, Chang HC. 2007. Production of gammaaminobutyric acid (GABA) by Lactobacillus buchneri isolated from kimchi and its neuroprotective effect on neuronal cells. J. Microbiol. Biotechnol. 17: 104-109. 
7. Wu Q, Shah NP. 2017. High $\gamma$-aminobutyric acid production from lactic acid bacteria: emphasis on Lactobacillus brevis as a functional dairy starter. Crit. Rev. Food Sci. Nutr. 57: 36613672.

8. $\mathrm{Wu} \mathrm{Q}$, Shah NP. 2015. Gas release-based prescreening combined with reversed-phase HPLC quantitation for efficient selection of high- $\gamma$-aminobutyric acid (GABA)-producing lactic acid bacteria. J. Dairy Sci. 98: 790-797.

9. Shan Y, Man C, Han X, Li L, Guo Y, Deng Y, et al. 2015. Evaluation of improved $\gamma$-aminobutyric acid production in yogurt using Lactobacillus plantarum NDC75017. J. Dairy Sci. 98: 2138-2149.

10. Small PL, Waterman SR. 1998. Acid stress, anaerobiosis and gadCB: lessons from Lactococcus lactis and Escherichia coli. Trends Microbiol. 6: 214-216.

11. Teixeira JS, Seeras A, Sanchez-Maldonado AF, Zhang C, Su MS-W, Gänzle MG. 2014. Glutamine, glutamate, and arginine-based acid resistance in Lactobacillus reuteri. Food Microbiol. 42: 172-180.

12. Azuma R, Ogimoto K, Suto T. 1962. Anaerobic culture method with steel wool. Nihon saikingaku zasshi. Jpn. J. Bacteriol. 17: 802-806.

13. Sanders JW, Leenhouts K, Burghoorn J, Brands JR, Venema G, Kok J. 1998. A chloride-inducible acid resistance mechanism in Lactococcus lactis and its regulation. Mol. Microbiol. 27: 299-310.

14. Feehily C, Karatzas K. 2013. Role of glutamate metabolism in bacterial responses towards acid and other stresses. J. Appl. Microbiol. 114: 11-24.
15. Yoshihashi T, Warun V, Patcharee T, Vipa S. 2009. Method for quantification of gamma-aminobutyric acid. Japan patent application PCT/JP2009/057537.

16. Cho S-Y, Han D-W, G-S L. 2018. GABA flat fish sikhae containing high GABA and method for preparing thereof. South Korea patent application KR20160077998.

17. Barrett E, Ross R, O'toole P, Fitzgerald G, Stanton C. 2012. $\gamma$-Aminobutyric acid production by culturable bacteria from the human intestine. J. Appl. Microbiol. 113: 411-417.

18. Ratanaburee A, Kantachote D, Charernjiratrakul W, Sukhoom A. 2013. Selection of $\gamma$-aminobutyric acid-producing lactic acid bacteria and their potential as probiotics for use as starter cultures in Thai fermented sausages (Nham). Int. J. Food Sci. Technol. 48: 1371-1382.

19. De Biase D, Pennacchietti E. 2012. Glutamate decarboxylasedependent acid resistance in orally acquired bacteria: function, distribution and biomedical implications of the gadBC operon. Mol. Microbiol. 86: 770-786.

20. Mazzoli R, Pessione E, Dufour M, Laroute V, Giuffrida MG, Giunta C, et al. 2010. Glutamate-induced metabolic changes in Lactococcus lactis NCDO 2118 during GABA production: combined transcriptomic and proteomic analysis. Amino Acids 39: 727-737.

21. Fernandez M, Zuniga M. 2006. Amino acid catabolic pathways of lactic acid bacteria. Crit. Rev. Microbiol. 32: 155183.

22. Zhuang K, Jiang Y, Feng X, Li L, Dang F, Zhang W, et al. 2018. Transcriptomic response to GABA-producing Lactobacillus plantarum CGMCC $1.2437^{\mathrm{T}}$ induced by L-MSG. PLoS One 13: e0199021. 